\title{
Modelling of Magneto-Elastic Phenomena in Inductive Dynamic Drive
}

\author{
Piotr Jankowski ${ }^{\dagger}$
}

\begin{abstract}
Inductive dynamic drives (IDD) are ultra rapid actuators where the moving part (disc) is subjected to impulse force. This paper presents the second model of inductive dynamic drive - a mechanical model where analytic- numerical approach was applied. The magnetic pressure, which was determined on the basis of the results obtained in the electrodynamic model, becomes the input data for mechanical model. Research with application of the mechanical model is necessary in order to determine the proper disc oscillation frequency and to obtain the stress state control for drive elements to be designed. Also, the selection of drive parameters to keep the disc deformation insignificant (while oscillating) is a condition under which these models do not need to be coupled together.
\end{abstract}

Keywords: Actuator, Magneto-elastic vibrations, Stresses

\section{Introduction}

The tested inductive-dynamic drive (IDD) is the main component of hybrid circuit breakers (Fig. 1.1), where the opening of contacts must take place at the appropriate time after the detection of a short circuit $[1,2,3]$. Therefore, the device must be characterized by huge dynamics (reaction time and acceleration) and repeatability so as to be able to synchronously work with semiconductor elements of such a switch. IDD is a system of a coil and a conductive disc where the coil is energized by a capacitor bank. As a result of the dynamic capacitor discharge, strong electrodynamic interaction takes place between the coil and the disc causing not only the movement of the disc but also its deformation. Therefore there was a need to create more accurate IDD model than hitherto, which would designate not only the distribution of electrodynamic forces acting on the disc [4], but also the mechanical behavior of the system including the stress distribution under the influence of these forces. A comprehensive model will allow for such a design of the physical actuator, which will operate in a reproducible manner which is a condition of a synchronous operation in a circuit breaker. It should be noted that in the proposed model the thermal phenomena were omitted due to the very short transitional character of current waveforms.

The main goals of the research presented in this article were: to carry out a study of radial forces proving their negligible influence on stretching of the plate (disc), to create a new mechanical model of IDD (MECH MODEL Fig. 1.2), in which analytic-numerical approach was applied. Analytical approach involves using the fact that the electrodynamic forces acting on the surface of the disc had cosinusoidal character and were approximated using such functions. In turn, the equation of vibration of the

$\dagger \quad$ Corresponding Author: Dept. of Marine Electrical Power Engineering, Gdynia Maritime University, Poland. (p.jankowski@we.am.gdynia.pl) Received: April 7, 2016; Accepted: December 12, 2016 thin plate subjected to axial forces while omitting radial components, was solved numerically, to carry out the stress analysis for the industrial actuator on the basis of a comprehensive model.

In the previous studies of IDD [5,6,7] such analyticnumerical approach has not been used, and this approach has the obvious advantage of significantly shortening the computation time. The computation time will be even more important if in the future there appears an idea of fully coupling electrodynamic model with a mechanical. A comprehensive model of IDD allows to precisely design its parameters such as the dimensions of the disc \& coil and also the initial voltage and capacitance of the capacitor supplying a coil so as not to exceed the allowable stress of the material used for the disc. Additionally, thanks to the MECH model, designer can and should control the differences between the frequency of extortion (electrodynamic force) and 1 th mode of disc vibration so as not to lead to resonance.

This paper is organized in the following manner: in chapter 2 radial forces occurring during operation IDD using the results of coil current, and eddy currents obtained from the existing electrodynamic model (ED Fig. 1.2) were designated. The ED model of this device was based on the

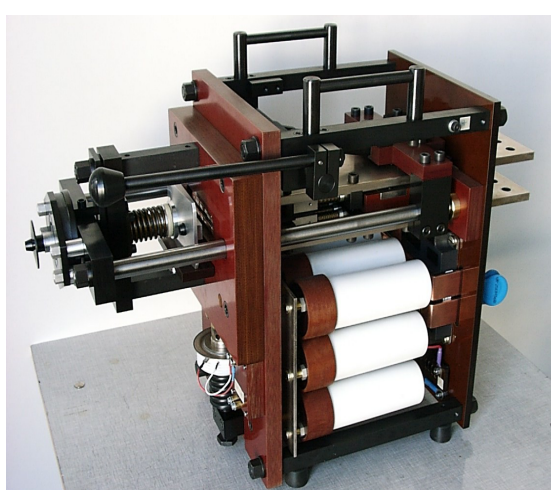

Fig. 1.1. Ultra rapid hybrid circuit breakers [3]. 


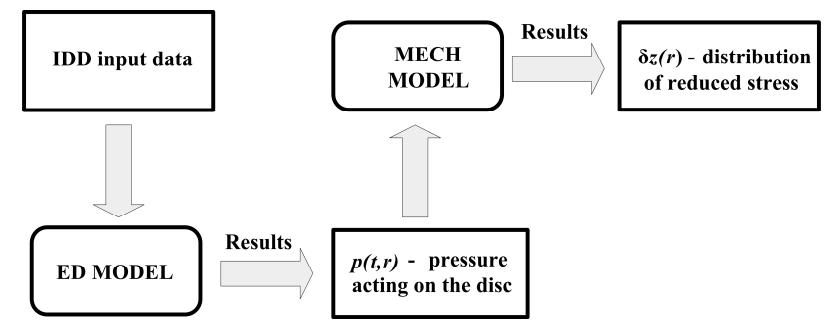

Fig.1.2. Block diagram for the reduced stresses calculation method for IDD disc.

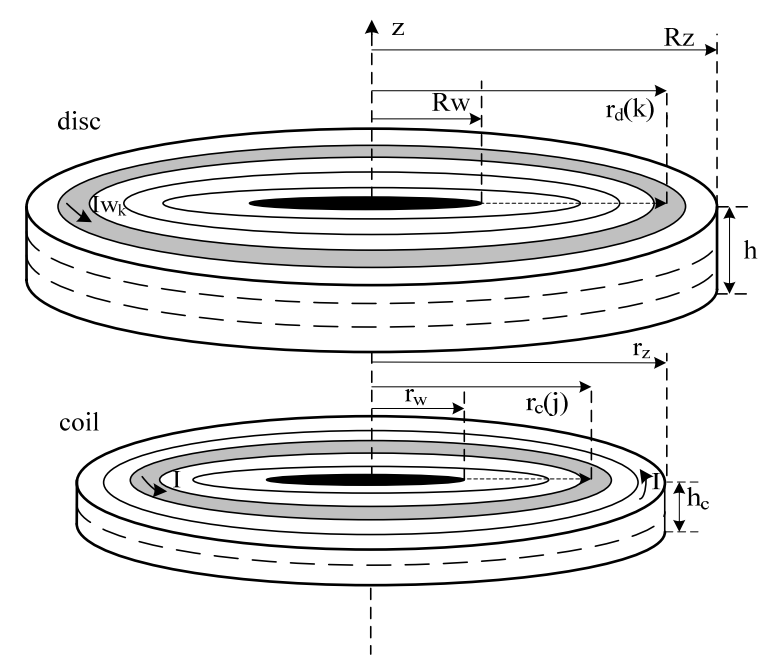

Fig.1.3. System of $n$ disc filaments and $k c$ coil sections

division of the disc into so-called filaments and of the coil into sections constituting the system of concentric circular loops (Fig.1.3). The method for quantitative determination of radial forces acting upon individual disc filaments for the selected system of coil and disc with application of the electrodynamic model (ED) is shown in chapter 2. Based on this model, it was found that the forces generated in the disc are concentrated in the first layer due to the skin effect. Therefore the mechanical model (MECH) and the result of simulations presented in this article are based on the solution of the thin plate equation (chapter 3 and 4). Excitation in this equation is the pressure resulting from the axial forces acting on the disc (Fig.1.2 and Fig.3.1). It was therefore necessary to examine whether the effect of radial forces occurring in the considered cases is negligible. The model describing electromagnetic phenomena (ED) was discussed in detail in [4].

\section{Electrodynamic Forces - Radial Componets}

Forces acting on the disc in the cylindrical coordinate system have two components: axial and radial (Fig.2.1). Radial forces do not have any influence on the axial disc movement. Those forces were also typically negligible in the strength analysis; researchers used to assume that those forces are of low values. Still, as the magnetic induction axial component tends to diffuse into the disc area, concerns grew that - under specific conditions - significant radial forces could be induced. If they were of a stretching character, a kind of disc stiffening would be caused, which could lead to significant modification of the stress distribution [8,9]. On the other hand, due to the vibrating disc movement, the radial force sense would have a stabilizing influence (with tension forces) or would cause greater deformation (when those forces are of compressing character - see Fig.2.1).

For the considered cylindrical system, induction components are related to the vector potential as in formulas:

$$
B_{r}=-\frac{\partial A_{\Theta}}{\partial z}, \quad B_{z}=\frac{A_{\Theta}}{r}+\frac{\partial A_{\Theta}}{\partial r}
$$

where

$$
\begin{aligned}
& A_{\Theta}=\frac{\mu_{0} I}{2 \pi} \sqrt{\frac{r_{c}}{r_{d}}}\left[\left(\frac{2}{k_{f}}-k_{f}\right) K\left(k_{f}\right)-\frac{2}{k_{f}} E\left(k_{f}\right)\right], \\
& k_{f}=2 \cdot \sqrt{\frac{r_{d} \cdot r_{c}}{\left(r_{d}+r_{c}\right)^{2}+(z)^{2}}}
\end{aligned}
$$

and $K\left(k_{f}\right), E\left(k_{f}\right)$ - respectively: first-order or second elliptical integer,

Therefore, analytical formulas which specify the magnetic field components (radial component and $z$ axis component) from a single current-conducting filament at any observation point have the following form:

$$
\begin{aligned}
& B_{r}(z)=-\frac{\mu_{0} I}{2 \pi} \sqrt{\frac{r_{c}}{r_{d}}}\left[\frac{z}{\left(r_{d}+r_{c}\right)^{2}+z^{2}}\left(\frac{2 K\left(k_{f}\right)}{k_{f}(z)}-\frac{\left(2-k_{f}(z)^{2}\right) E\left(k_{f}\right)}{\left(1-k_{f}(z)\right)^{2} k_{f}(z)}\right)\right] \\
& B_{z}(z)=\frac{\mu_{0} I}{2 \pi} \frac{1}{\sqrt{z^{2}+\left(r_{c}+r_{d}\right)^{2}}}\left[K\left(k_{f}\right)+\frac{-z^{2}+r_{c}^{2}-r_{d}^{2}}{z^{2}+\left(r_{c}-r_{d}\right)^{2}} E\left(k_{f}\right)\right]
\end{aligned}
$$

The radial force acting upon the $k$-th disc filament was divided into three elements:

$$
F r_{k}=F w r_{k}+F c r_{k}+F p r_{k}
$$

where: $F w r_{k}$ is the radial force acting upon the $k$-th filament and originating from the field created by the remaining filaments. The force formula is as follows:

$$
F w r_{k}=2 \pi r_{d}(k) I w_{k} \cdot \sum_{j=1 \wedge j \neq k}^{n} B_{z}\left(z, r_{d}(k), r_{d}(j), I w_{j}\right)
$$

$F c r_{k}$ is the radial force acting upon the $k$-th filament and 
originating from the field created by the number of $k_{c}$ sections of the coil containing $z_{w}$ windings each. This force is described as follows:

$$
F c r_{k}=2 \pi r_{d}(k) I w_{k} \frac{z_{w}}{k_{c}} \sum_{j=1 \wedge j \neq k}^{k_{c}} B_{z}\left(z, r_{d}(k), r_{c}(j), I_{c o i l}\right)
$$

$F p r_{k}$ is the radial tension force acting upon the $k$-th filament and originating from the current of that filament:

$$
F p r_{k}=\mu_{0} \frac{\left(I w_{k}\right)^{2}}{2}\left[\ln \left(8 \frac{r_{d}(k)}{r_{o}}\right)-0.75\right]
$$

Simulations were made for the drive applied to an ultrahigh-speed AC breaker [3]. Parameters for that drive are included in Table 1.

For radial forces tests, the force sense towards the outside of the winding (tear force) was adopted as 'negative', whereas the compression force sense was adopted as positive. Due to the strong skin effect being present for the adopted drive parameters, both the eddy current density and the forces (which resulted from eddy currents) acting along the thickness of the disc, faded quickly. That observation is valid for both the radial component and axial component of the force. That is why,

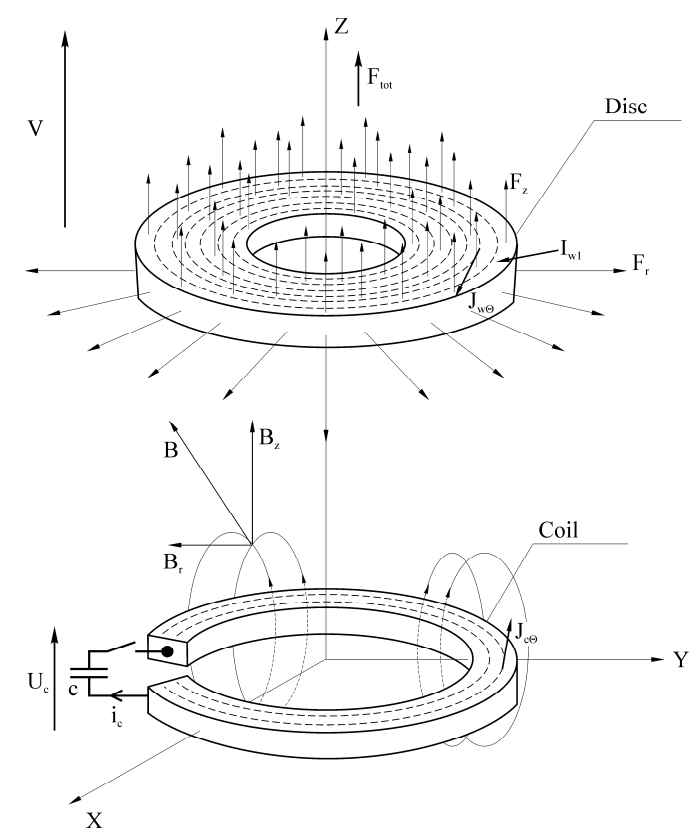

Fig.2.1. Force components acting upon the disc filaments

Table 1. Parameters of IDD for radial force tests

\begin{tabular}{c|c}
\hline $\begin{array}{c}\text { Initial energy of capacitor: } \\
E=1250 \mathrm{~J}\end{array}$ & Capacitance of capacitor: $C=100 \mu \mathrm{F}$ \\
\hline Outer radius of disc: $R z=5.5 \mathrm{~cm}$ & Outer radius of coil: $r_{z}=5.5 \mathrm{~cm}$ \\
\hline Inner radius of disc: $R w=2.75 \mathrm{~cm}$ & Inner radius of coil: $r_{w}=3 \mathrm{~cm}$ \\
\hline Add mass: $0.31 \mathrm{~kg}$ & Winding of coil: $z_{w}=11$ \\
\hline $\begin{array}{c}\text { Materials of disc: } \\
\text { duraluminium, } h=4 \mathrm{~mm}\end{array}$ & Thickness of coil: $h c=1.1 \mathrm{~cm}$ \\
\hline
\end{tabular}

in the further discussion, the radial pattern will be shown for the disc's first layer (or skin depth) according to the division into layers as in the electrodynamical model. Fig. 2.2 shows examples of radial forces acting upon selected filaments and which originated from fields of the remaining filaments $\left(F w_{r}\right.$ element). Like axial forces, those forces are generally single-direction ones, with a tear action on internal filaments, and compression action on external filaments. Forces acting on individual filaments and which originated from the coil's magnetic field were similar to those in Fig. 2.2. But this time, the forces acting on internal filaments were of a compressive character, and those acting on external filaments were of a tearing character. The location where the components reverse their senses depends on the coil/disc radius ratio. Fig. 2.3 shows the sum of all three elements, or in other words resultant radial forces acting upon individual filaments.

As could be predicted, elements of radial force compensate each other in part only. Yet, in the central disc part, radial forces are of an alternate character against time (which means tearing and compressing alternately). Fig. 2.4 shows the force distribution along the disc radius for

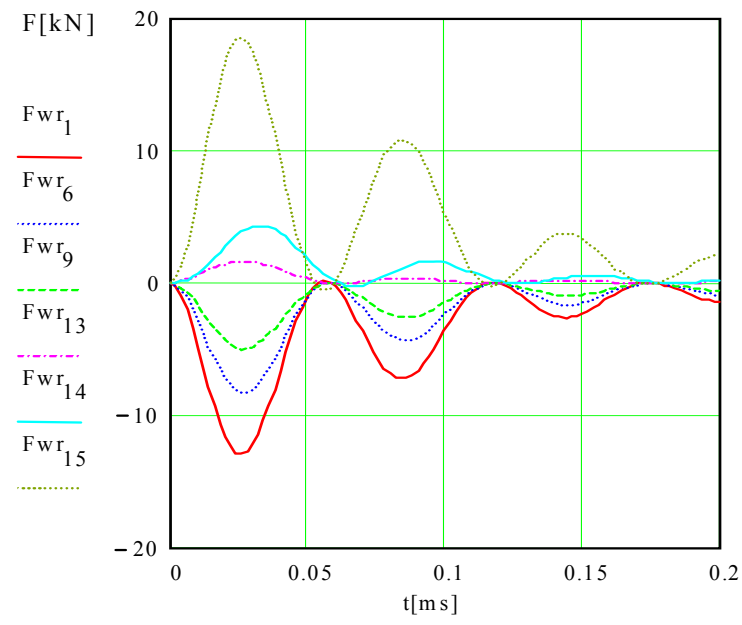

Fig.2.2. Fwr forces acting on selected disc filaments

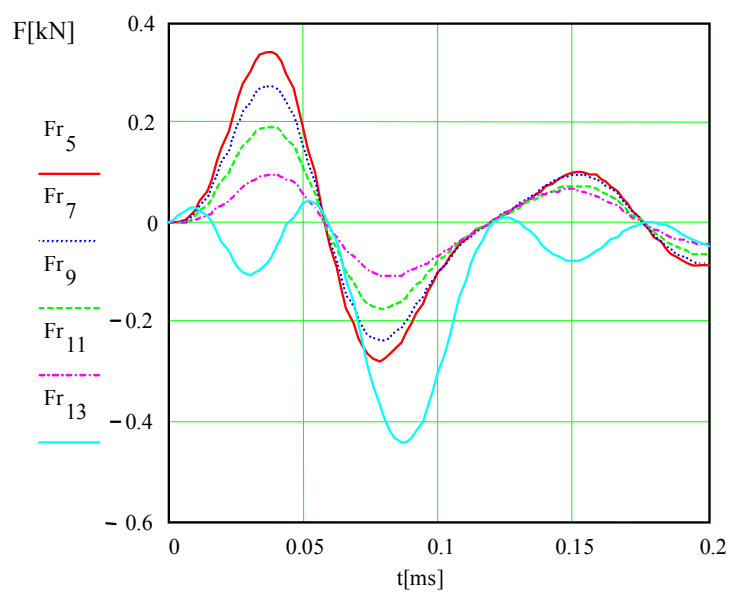

Fig.2.3. $\mathrm{Fr}$ forces acting on selected disc filaments 


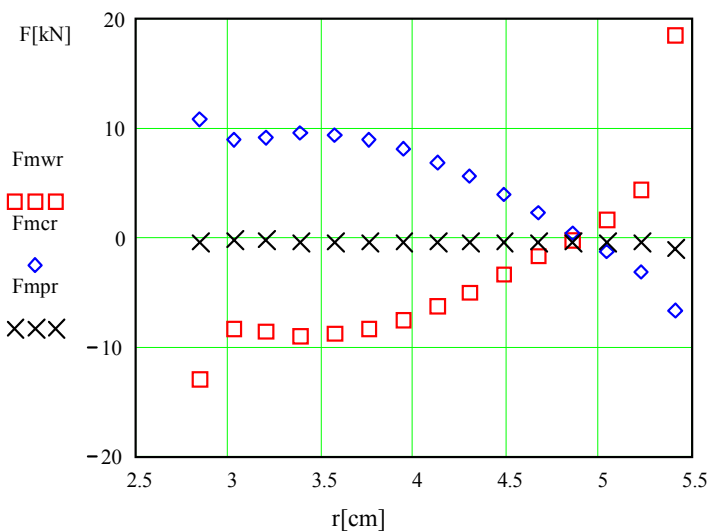

Fig.2.4. Suprems (infims) of radial force elements

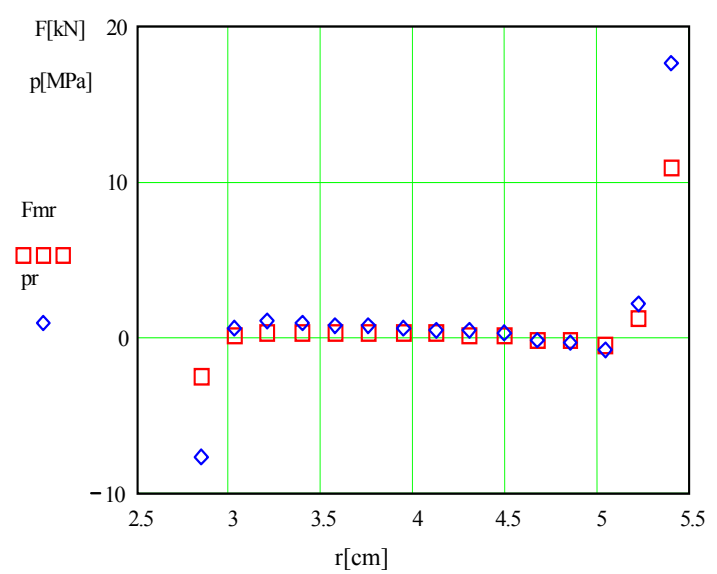

Fig.2.5. Suprem (infim) of total radial forces and pressures

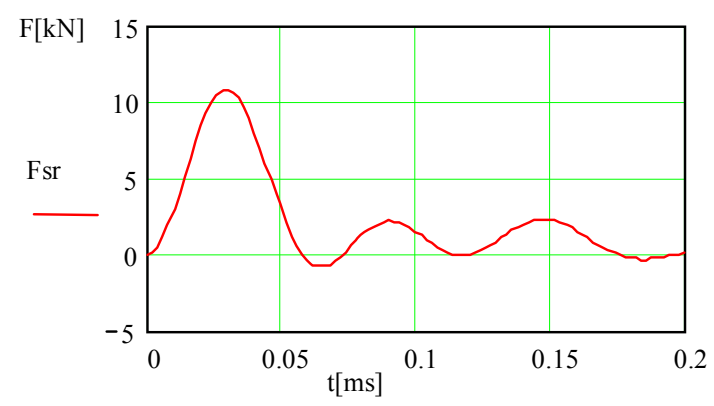

Fig.2.6. Total radial force upon a disc

the maximum (or minimum) radial force values. An observation can be made that in filaments, radial forces originating from the filament's own current have small values compared to other elements $\left(F m p r_{i}<0.2 \mathrm{kN}\right)$. Fig. 2.5 shows the distribution of suprems (or infims) for the resultant radial force (sum of all elements) and of pressures acting upon individual filaments. As can be seen, the greatest forces act on the external disc filaments. Besides them, the largest resultant value did not exceed $1.5 \mathrm{kN}$, that figure being equivalent to the pressure of $2 \mathrm{MPa}$ acting upon the filament side surface. The total sum of all forces acting on all filaments determines the total radial force

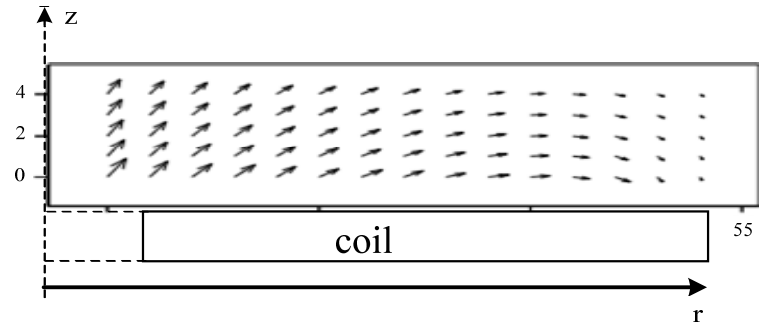

Fig.2.7. Magnetic field force lines for the coil without with co-interaction from the disc current

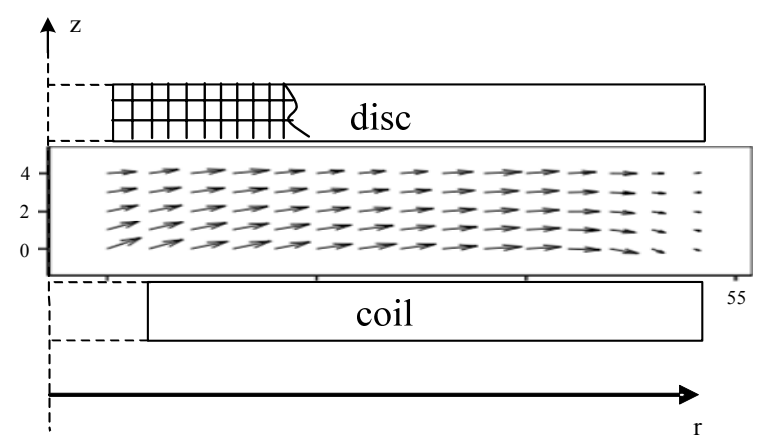

Fig.2.8. Magnetic field force lines in the gap $(4 \mathrm{~mm})$ between coil and disc

acting on the disc (Fig.2.6). In the case discussed here, this force is practically of a compressive character all the time. During simulation tests with coil and disc systems featuring different radial dimensions, cases were observed with the radial force having an alternate character (tensile for the first pulse, compressive for the second pulse).

The reason for compensation of investigated radial force components originated from the magnetic field of the coil and the eddy current of the disc is explained by the magnetic field pattern in the gap between the coil and the disc. Fig. 2.7 shows the simulation determining magnetic field lines (vector field) around the coil with no eddy current influence, whereas Fig. 2.8 shows the image of force lines when considering this moment when the disc has reached a distance of $4 \mathrm{~mm}$ from the coil. The field force line image supports the conclusion that the magnetic flux density axial component $B_{z}$, has a negligible value when the disc is $4 \mathrm{~mm}$ from the coil. For greater distances, field pattern examinations proved that the magnetic field $B_{z}$ component (responsible for radial forces) is of an insignificant value, this being a result of the quickly fading eddy currents and coil current due to the transient character of the phenomenon.

Tests made for different coil current pulsations, and with the same initial energy from the capacitor battery, showed that radial forces increased insignificantly for central filaments, but on the other hand, maximum force values decreased for extreme filaments. For instance: for the capacitor battery enlarged 50 times in capacity and with the voltage reduced accordingly to obtain the same initial energy $(E=1250 \mathrm{~J})$ as before, the maximum force value 


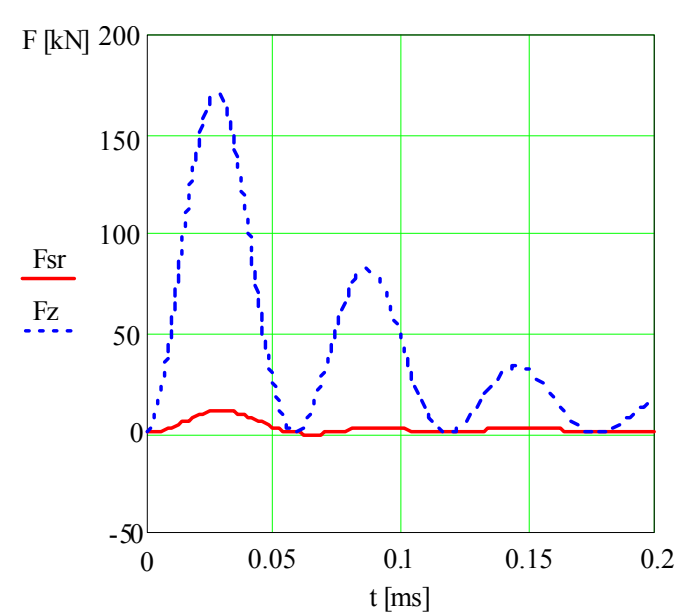

Fig.2.9. Comparison of total radial and axial forces acting on the disc

did not exceed $2 \mathrm{kN}$ for extreme filaments as well. Radial force pattern examinations for upper disc layers confirmed that all force components decreased dramatically, radial forces included.

When comparing the complete radial force and axial force acting on the disc (Fig. 2.9), it was found (for all cases tested) that the first of them had a significant overbalance. Therefore, it seems that the application of the thin plate vibration equation for the mechanical model (MECH), omitting the influence of the radial forces on the disc stiffness, is substantiated in the given application range. 4

\section{Mechanical Model (mech) Analytic Numerical Approach}

In order to obtain the required acceleration (in practice, more than $100,000 \mathrm{~g}$ ), an appropriately large force/mass ratio should be ensured for the drive. A mass reduction by changing the radial dimensions (e.g. reduction of the disc external radius below the coil radius) results directly in reducing the induced force. On the other hand, when the disc thickness is reduced, the force decreases significantly no earlier than when the thickness is similar to the skin depth value. The ED tests demonstrated that for coil current frequency $>20 \mathrm{kHz}$, the skin layer thickness (for aluminium discs as tested) was small and $<2 \mathrm{~mm}$ [10]. Therefore, in order to obtain a maximum force/mass ratio, the disc thickness should be slightly larger than the skin layer thickness. Yet, it should be kept in mind that an insufficient disc thickness can be the reason for a permanent deformation thereof. In practice, the disc radius/thickness ratio meets the condition :

$$
h \leq a \cdot\left(R_{z}-R_{w}\right)
$$

where: $h$-disc thickness and $a \in(0.1 \div 0.2)$, which is also

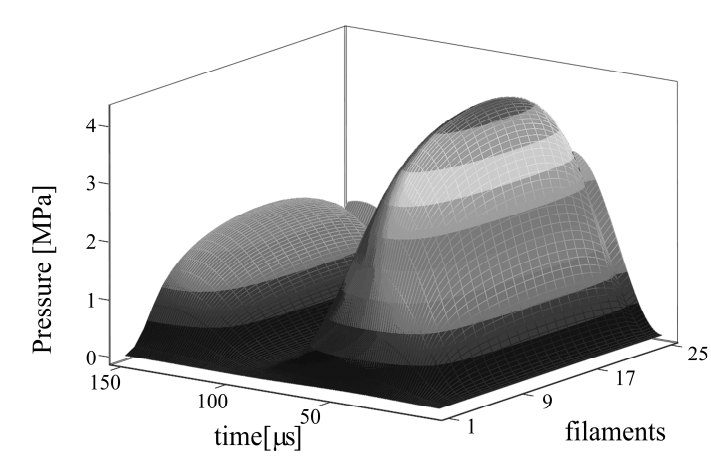

Fig.3.1. Time-spacing distribution of the pressure $p(r, t)$ obtained from ED

the applicability condition for the thin plate equation [11].

For a uniform plate of constant thickness $(D=$ const) and taking into consideration the cylindrical symmetry of plate and extortion, equation of a thin plate movement has the following form (3.1):

$$
\frac{\partial^{2} w}{\partial t^{2}}+\frac{D}{\bar{\rho}} \frac{1}{r} \frac{\partial}{\partial r}\left\{r \frac{\partial}{\partial r}\left[\frac{1}{r} \frac{\partial}{\partial r}\left(r \frac{\partial w}{\partial r}\right)\right]\right\}=\frac{p(r, t)}{\bar{\rho}}
$$

where: $\bar{\rho}=h \rho$ - plate mass per unit area, $w=w(r, \varphi, t)$ - displacement, $D=\frac{E h^{3}}{12\left(1-v^{2}\right)}-$ flexural rigidity,

$E, h, v$-respectively: Young modulus, plate thickness, Poisson ratio,

$p(r, \varphi, t)$ - pressure distribution against plate surface and time (Fig. 3.1).

Separation of variables was applied to solve this problem. The particular integrals are presented in the following form:

$$
w_{i}(r, t)=W_{i}(r) T_{i}(t)
$$

By substitution of (3.2) to the homogeneous form of equation (3.1), the following equation system is obtained:

$$
\begin{gathered}
\ddot{T}_{i}+\omega_{i}{ }^{2} T_{i}=0 \\
\frac{1}{r} \frac{d}{d r}\left\{r \frac{d}{d r}\left[\frac{1}{r} \frac{d}{d r}\left(r \frac{d W_{i}}{d r}\right)\right]\right\}=\lambda_{i}{ }^{4} W_{i}
\end{gathered}
$$

where: $\lambda_{i}{ }^{4}=\frac{\omega_{i}^{2} \bar{\rho}}{D}$.

The solution for Eq. (3.4), which meets the boundary conditions, consists of so-called eigenfunctions, $W_{i}$, and eigenvalues, $\lambda_{i}$ corresponding to those eigenfunctions. Free disc vibration frequencies $\omega_{\mathrm{i}}$ are related to the eigenvalues. Because the solution for non-homogeneous equation (3.1) 
is expected to have the following form:

$$
w(r, t)=\sum_{i=0}^{\infty} T_{i}(t) W_{i}(r)
$$

The extortion has the form of:

$$
p(r, t)=\sum_{i=0}^{\infty} \Phi_{i}(t) W_{i}(r)
$$

After substitution of (3.5) and (3.6) to (3.1) and after reseparation of variables, two equations are obtained. The solution for the first equation consists of the eigenfunctions (defined already). The solution for the second equation has the following form:

$$
\ddot{T_{i}}+\omega_{i}^{2} T_{i}=\frac{1}{\bar{\rho}} \Phi_{i}
$$

Eigenfunction orthogonality is applied for determination of coefficients for the sequence of numbers.

Because:

$$
\int_{r_{0}}^{n_{k}} p(r, t) r W_{j}(r) d r=\sum_{i=0}^{\infty} \Phi_{i} \int_{r_{0}}^{r_{k}} r W_{i}(r) W_{j}(r) d r
$$

and:

$$
\int_{r_{0}}^{n_{k}} r W_{i} \cdot W_{j} d r=\left\{\begin{array}{c}
\gamma_{i}^{2} \text { for } i=j \\
0 \text { for } i \neq j
\end{array}\right.
$$

therefore, functions $\Phi_{i}$ corresponding to the consecutive eigenfunctions and eigenvalues are determined by integers.

$$
\Phi_{i}=\frac{1}{\gamma_{i}^{2}} \int_{r_{0}}^{n_{k}} p(r, t) r W_{i}(r) d r
$$

Observing the resultant forces acting on individual plate filaments (Fig. 3.2), their cosine character and the varying widths of consecutive pulses can be noticed. Based on that

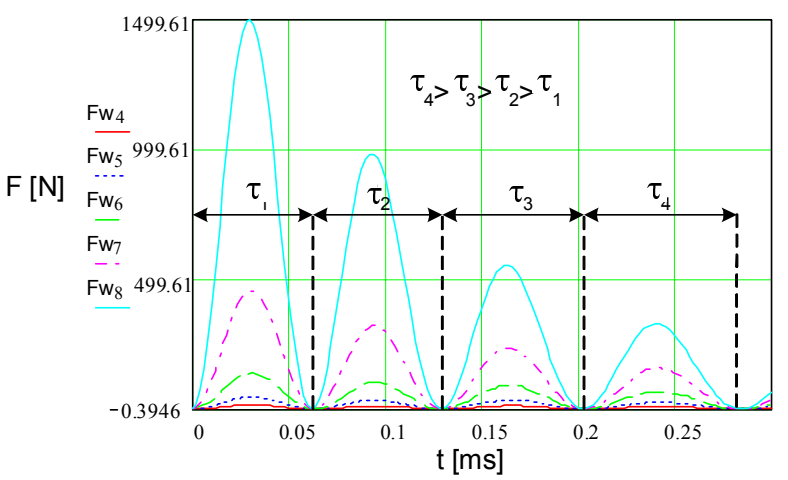

Fig.3.2 Force upon selected disc filaments feature, the pressure curve for the $k$-th filament was approximated with application of the following analytical function:

$$
p_{k}(t)=\chi_{j} \frac{p_{m k}}{2}\left(1-\cos \frac{2 \pi}{\tau_{j}} t\right)
$$

For pressure acting upon the $k$-th filament, and with application of approximation (3.11), the extortion $p(r, t)$ is described by the function as follows:

$$
p(r, t)=\left\{\begin{array}{cl}
0 & r<(k-1) s \\
p_{k}(t) & (k-1) s \leq r \leq k s \\
0 \quad k s \leq r
\end{array}\right.
$$

where: $s$ - filament width

Therefore, for a single filament, the $\Phi$ function has the following form:

$$
\Phi_{i j k}=\frac{\chi_{j} \frac{p_{m k}}{2}\left(1-\cos \frac{2 \pi}{\tau_{j}} t\right)}{\gamma_{i}^{2}} \int_{(k-1) s}^{k s} r W_{i}(r) d r
$$

Formula (3.7) takes the following form:

$$
\frac{d^{2} T_{i j k}}{d t^{2}}+\omega_{i}^{2} T_{i j k}=c_{i j k}\left(1-\cos \frac{2 \pi}{\tau_{j}} t\right)
$$

where: $c_{i j k}=\frac{\chi_{j} p_{m k}}{2 \gamma_{i}^{2}} \int_{(k-1) s}^{k s} r W_{i}(r) d r$

and $\quad i=0,1,2 \ldots . l_{f}-$ oscillation form indicator,

$j=0,1,2 \ldots . l_{t}-$ load period indicator,

$k=0,1,2 \ldots . l_{n}-$ disc filament indicator.

In case of loading numerous filaments, $l_{n}$ being their number, this differential equation can be solved $l_{n}$ times and $i$ times for specified eigenvalues. The solution for the entire disc load is treated as a superposition of solutions for separate filaments. In case of further $j$ force pulses, whose period $\tau_{j}$ varies, the differentiation operation should be done $j$ times taking into consideration - for each new impulse - new initial conditions as determined for the previous case. That was the way to obtain solutions for a non-homogeneous equation (3.1) with specified boundary conditions and 'zero' initial conditions. This solution describes the location of central surface for each position and time point as follows:

$$
w(r, t)=\sum_{i=0}^{l f} \sum_{k=1}^{l p} W_{i}(r) T_{i j k}(t)
$$

When obtained that way, the solution facilitates stress 
determination for any disc point at any time during the process. The stresses are selected in compliance with the effort hypothesis as chosen. Among numerous existing effort hypotheses, the shape deformation energy hypothesis, also called the Huber-Mises-Henecky hypothesis, is ranked

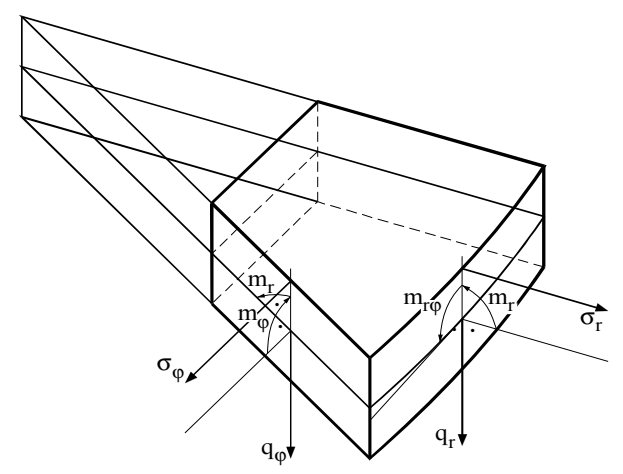

Fig.3.3. Normal stresses and bending moment in circular sector

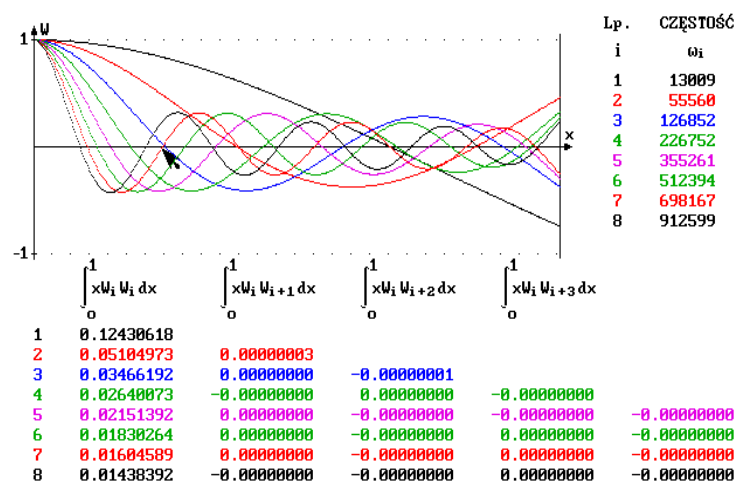

Fig.3.4. First screen of programm $\mathrm{MECH}$, with finding eigen functions (parameters in Table 1)

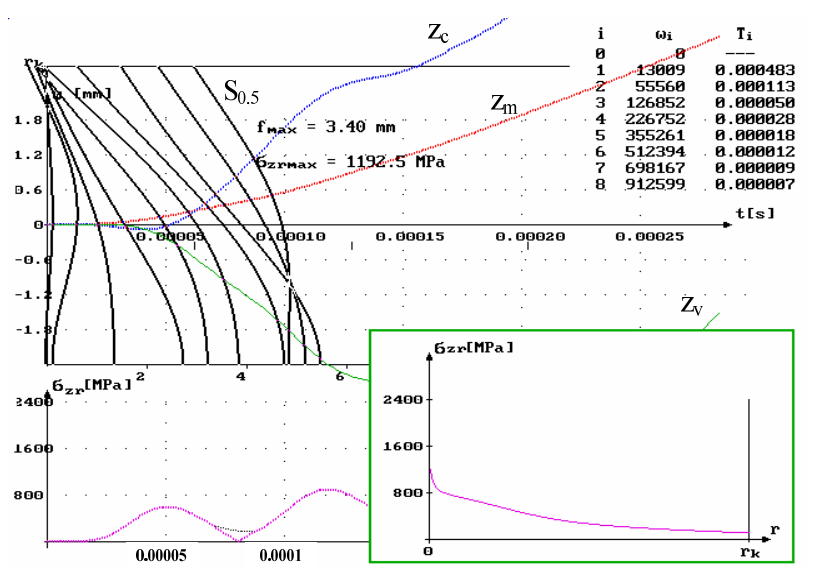

Fig.3.5. Screenshot of the program solving movement equation for disc vibration and for the state of stress (for data in Table 1). $S_{0.5}$ - deformed central surface for selected time points, $Z_{m}$ - centre of the mass displacement, $Z_{c}$ - disc centre displacement, $Z_{V}$ - disc oscillations with relation to the centre of the mass, $\sigma_{z r}$ - reduced stresses vs. time, Subscreen - curve for maximum $\sigma_{z r}$ values vs. radius among the most frequently applied. In order to estimate the material effort, the notion of equivalent reduced stress $\sigma_{\text {red }}$, is introduced. According to the material effort hypothesis, the equivalent reduced stress equation has the form of:

$$
\sigma_{r e d}=\sqrt{\sigma_{r}^{2}+\sigma_{\varphi}^{2}-\sigma_{r} \sigma_{\varphi}}
$$

where: $\sigma_{r}, \sigma_{\phi}$ - normal stresses as in Fig. 3.3.

The mathematical algorithm for the MECH model was made in Delphi language as application for which the same name (MECH) was assumed as that of the model. Calculations are carried out in two stages. Stage 1 consists in determination of eigenfunctions and eigenvalues, and their orthogonality is checked (Fig. 3.4).

In stage 2 , the software determines the movement trajectory (displacement for the centre of the mass and for the disc) and the maximum reduced stress within the disc (Fig. 3.5)

As seen in Fig. 3.5, the software also shows animations for disc surface movement (half of disc). As an option, a window can be opened containing the maximum reduced stress distribution vs. radius. This distribution confirms that the highest stress value is that in the disc centre.

\section{Stress analysis}

The basic requirement for IDD is to achieve the greatest acceleration possible (in practice, travel a required distance over a required time). At the same time, disc stress must not exceed the maximum allowable value, or else a permanent disc deformation is possible. A majority of IDD parameters have an influence on the acceleration and stress. Those parameters include $R, L$ and $C$ of the coil circuit, and longitudinal and transverse dimensions of both coil and disc. The optimization issue could consist in minimization of the multi-dimension functional. In practice, radial dimensions of such a system are limited due to design limits of the object containing the IDD (such as breaker), [3]. In turn, minimizing the $R, L$ and $C$ parameters is the reason for increasing the system dynamic properties. The disc thickness has an influence on the resultant interaction force, and this influence will decrease as soon as the disc thickness exceed the skin depth. Hence, excessive disc thickness results in a decrease of the acceleration due to the disc gaining mass quicker than the force increases. On the other hand, the significant influence of the disc thickness on the resultant stress should be kept in mind. Therefore, the disc thickness should be slightly greater than the skin layer thickness - assuming that the maximum stress will not exceed the allowable limit value. Fig. 4.1 shows the maximum reduced stress and deviations vs. disc thickness for the drive parameters as shown in Table 2, which were obtained by MECH software simulation. Because the external pressure acting on the disc is the extortion in the thin plate vibration equation, forces obtained within the 
Table 2. Parameters of IDD for $\mathrm{h}=\mathrm{variab}$

\begin{tabular}{c|c}
\hline Initial energy of capacitor: $E=450 \mathrm{~J}$ & Capacitance of capacitor: $C=100 \mu \mathrm{F}$ \\
\hline Outer radius of disc: $R z=8 \mathrm{~cm}$ & Outer radius of coil: $r_{z}=6 \mathrm{~cm}$ \\
\hline Inner radius of disc: $R w=0 \mathrm{~cm}$ & Inner radius of coil: $r_{w}=2.4 \mathrm{~cm}$ \\
\hline Add mass: $0 \mathrm{~kg}$ & Winding of coil: $z_{w}=12$ \\
\hline $\begin{array}{c}\text { Material: duraluminium } \\
h=4,6,8,10,12,14 \mathrm{~mm}\end{array}$ & Thickness of coil: $h c=0.3 \mathrm{~cm}$ \\
\hline
\end{tabular}

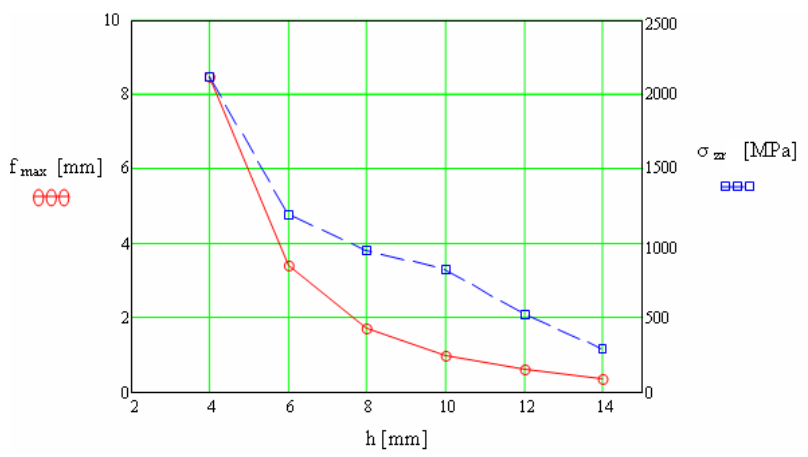

Fig.4.1. Results of maximal $\sigma_{\mathrm{zr}}$ and deviation for various thickness of the disc

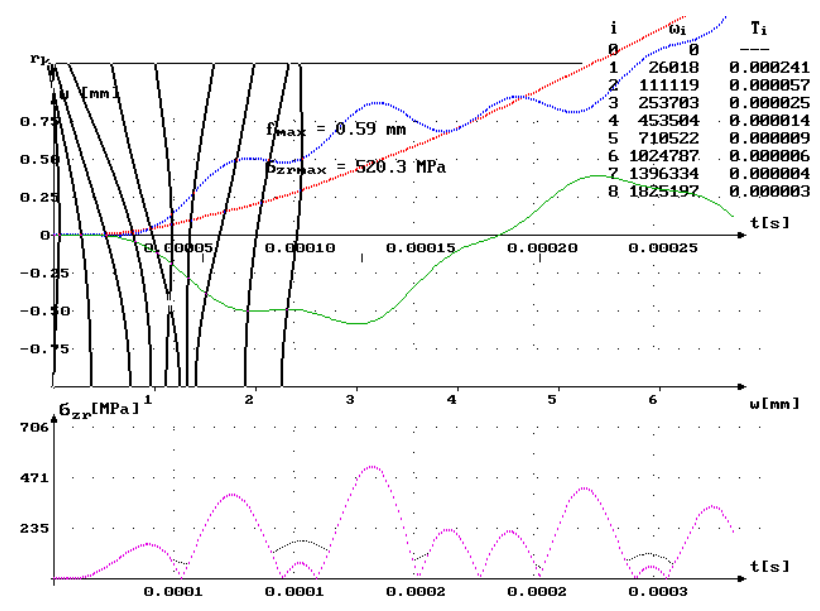

Fig.4.2 Result for thickness of disc $h=12 \mathrm{~mm}$ (descripton as in Fig. 3.5)

entire disc volume were added appropriately (along the disc thickness). As said before, that operation is even more substantiated for higher current frequencies as resulting from $R, L$ and $C$ values, which reduces the skin layer depth. For the case discussed here, the reduced stresses (maximum) exceeded $520 \mathrm{MPa}$ even for the disc thickness $=12 \mathrm{~mm}$ (Fig.4.2). That value is higher than the allowed reduced stress of $500 \mathrm{MPa}$ for the aluminium alloy as applied. Nevertheless, it should be emphasised that the pressure pattern depends on the disc/coil dimension ratio, and further, this pressure pattern has a significant influence on the stress distribution.

Until recently, assumptions were made that the radial coil dimension should be significantly smaller in order to obtain optimum coupling between the coil and the disc. For instance, references $[12,13]$ stated that the best coil/disc

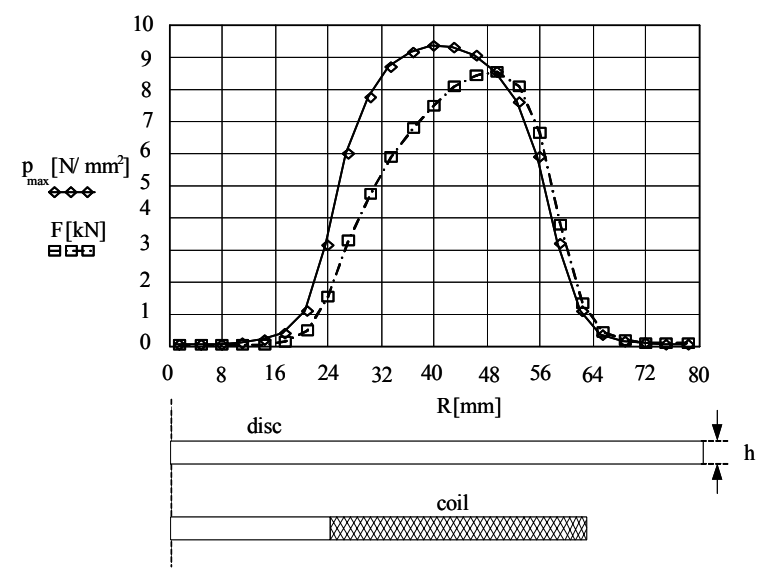

Fig.4.3. Distribution of the pressure and force for the data taken from Table 1 .

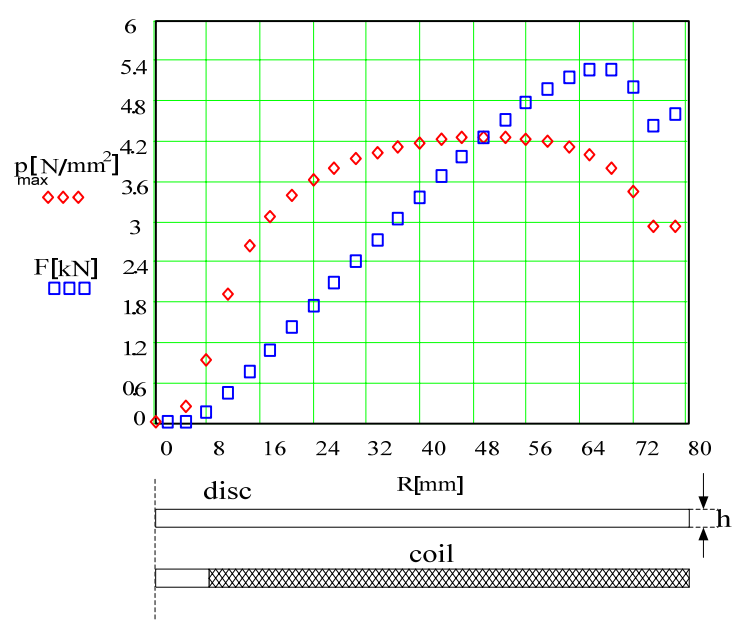

Fig.4.4. Distribution of pressure and force for wider coil

external radius ratio is 0.75 . Yet, based on a comparison of the pressure pattern in Fig. 4.3 against that in Fig. 4.4 (obtained for the same parameters as in Table 2 excluding coil radiuses of $\mathrm{r}_{w}=0.8 \mathrm{~cm}$ (internal radius) and $\mathrm{r}_{z}=8 \mathrm{~cm}$ (external radius), a more uniform pressure pattern can be observed.

The effect thereof consisted in a dramatic reduction of the maximum reduced stress: from more than $2000 \mathrm{MPa}$ (Fig.4.1) to $250 \mathrm{MPa}$ (for a full disc of $\mathrm{h}=4 \mathrm{~mm}$ thickness). Tests carried out for discs and for system parameters as in Table 2 resulted in confirmation for permanent disc deformations for $h<10 \mathrm{~mm}$.

\section{Conclusion}

The inductive-dynamic drive must have a high repeatability of the process. Only then does it ensure proper cooperation as a main part of hybrid circuit breaker. Perfect repeatability is also necessary in the case of synchronous operation of several drives used in multiphase systems [14]. 
On the other hand, the set drive parameters must ensure appropriate high dynamics of the disc. This means that the maximum force with its most uniform distribution along radius should act on the disc. Then, even the plastic deflections of the disc will be slight. That is why it seems that application of a separate electro-dynamic model, with results thereof being input data for the mechanical model, would facilitate determination of IDD structural parameters to meet actual requirements. Mechanical model tests do allow not only the stress state determination for designed IDD elements but also make it possible to control the necessary difference between the frequency of induced force and the disc's natural vibration frequency. Other studies (not presented in this paper) showed a dramatic increase in stress when those frequencies were similar to each other (resonance) $[10,13]$. The analyticalnumerical approach as in ED and MECH models can be used to calculate optimum disc dimensions, which means: dimensions facilitating a required displacement over a required length of time with the least initial capacitor energy possible as well as below the limit value for the reduced stress.

\section{References}

[1] A.M.S Atmadji, and Sloot J.G.J, "Hybrid Switching a Review of Current," In Proceedings of the International Conference on Energy Management and Power Delivery, EMPD 2: 683-688. doi:10.1109/ EMPD.1998.702771.

[2] D. D. Shipp, T. J. Dionise, V. Lorch, and W. G. MacFarlane, "Vacuum Circuit Breaker Transients During Switching of an LMF Transformer," IEEE Trans. Ind. Appl., vol. 48, no. 1, pp. 37-44, Jan./Feb., 2012.

[3] J. Czucha, P. Jankowski, M. Pikoń, J. Zyborski, "Ultra rapid contact member with arcless current commutation" In Proceedings of the International Conference on Electrical Contacts, Electro-mechanical Components and their Applications ICECT'99," July 19-24 1999, Nagoya.

[4] P. Jankowski "Analytic-numerical approach in modelling electrodynamic phenomena of inductive dynamic drive," Journal of the Chinese Institute of Engineers, 2015 http://dx.doi.org /10.1080/02533839 2015.1070688.

[5] Xiu-xing Yin, Yong-gang Lin, Wei Li, Ya-jing Gu, Peng-fei Lei, Hong-wei Liu. "Sliding Mode Voltage Control Strategy For Capturing Maximum Wind Energy Based on Fuzzy Logic Control," International Journal of Electrical Power \& Energy Systems. 2015, 70, 45-51.

[6] Xiu-Xing Yin, Yong-Gang Lin, Wei Li, Hong-Wei Liu, and Ya-Jing Gu. Fuzzy-logic "Sliding-Mode Control Strategy for Extracting Maximum Wind
Power," IEEE Transactions on Energy Conversion. 2015, 30(4), 1267-1278.

[7] J. S. Ro, S. K. Hong, and H. K. Jung, "Characteristic Analysis and Design of a Novel Permanent Magnetic Actuator for a Vacuum Circuit Breaker," Electric Power Applications, IET, vol. 7, no. 2, pp. 87-96, Feb., 2013.

[8] Kim Won-Ho, "A Stress Analysis Method for the Rotor Design of an IPMSM Considering Radial Force," Journal of Electrical Engineering \& Technology Vol.; 11(2): 377-382, 2016.

[9] Lee Gyeong-Deuk, Kim Gyu-Tak "The Equilibrium Design of Radial Magnetic Force for Reduction" of Vibration in IPM Type BLDC Motor, Journal of Electrical Engineering \& Technology Vol. 9, No. 3, pp. 882-887, 2014

[10] P. Jankowski, J. Mindykowski., M. Woloszyn.: "Effect of Power Frequency on the Stress State of Disc Actuator," International Journal of Applied Electromagnetics and Mechanics Volume 45, Numbers 1-4 IOS Press doi:10.3233/JAE-141888.

[11] S. Timoshenko and S. Wojnowski, Theory of plates and shells. New York and London: Mcgraw-Hill, 1959.

[12] S. Basu, and K.D. Srivastava, "Analysis of A Fast Acting Circuit Breaker Mechanism Part I: Electrical Aspects," Transaction Power Apparatus and Systems 1972, PAS-91 (3): 1197-1203. doi:10.1109/TPAS. 1972.293477.

[13] S Basu, and K.D. Srivastava, "Analysis of A Fast Acting Circuit Breaker mechanism Part II: Thermal and Mechanical Aspects," Transaction Power Apparatus and Systems, 1972 PAS-91 (3): 1203-1211. doi:10.1109/TPAS.1972.293478.

[14] P. Mysiak, "A 24-pulse Diode Rectifier with Coupled Three-Phase Reactor," Journal of the Chinese Institute of Engineers, 200730 (7): 1189-1204. doi:10.1080/ 02533839.2007 .9671347 .

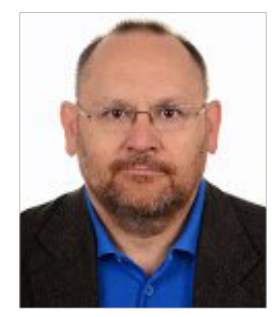

Piotr Jankowski Assistant Professor at the Department of Marine Electrical Power Engineering, Gdynia Maritime University. Born on October 28, 1961 Zabrze, Poland. Graduated with a M.Sc. degree in Electrical Engineering from Silesian University of Technology in1986. In 1998 he received his $\mathrm{Ph} . \mathrm{D}$ in electrical engineering from Gdansk University of Technology. An author of numerous research publications and reports on mathematical modelling of conjugate fields. 